\title{
Applications of Surface Correlation to the Estimation of the Harmonic Fundamental of Speech
}

\author{
Douglas J. Nelson \\ R523, U.S. Department of Defense, Ft. Meade, MD 20755, USA \\ Email:waveland@erols.com \\ Received 24 July 2001
}

\begin{abstract}
We present a method for estimating the fundamental frequency of harmonic signals, and apply this method to human speech. The method is based on cross-spectral methods, which provide accurate resolution of multicomponent FM signals in both time and frequency. The fundamental is re-introduced to the spectrum by a frequency-lag autocorrelation of the spectrum, even if the fundamental is completely missing in the original spectrum. By combining the different perspectives of the Fourier spectral representation and the time-lag autocorrelation function we suppress all components of harmonic signals except for the fundamental.
\end{abstract}

Keywords and phrases: cross-spectrum, phase spectrum, STFT, Fourier transform, speech, formant recovery, equalization.

\section{INTRODUCTION}

In applications, such as speech vocoding or tasks, such as determining the identity or gender of the speaker of a short segment of speech, accurate estimation of excitation and formants (vocal tract resonances) is an important problem. The basic structure of voiced speech is the superposition of vocal tract resonances, which are excited by a quasi-periodic train of pulses formed at the glottis at the back of the vocal tract. While both the excitation frequency $\omega_{0}$ and the formant frequencies are nonstationary, it is well known that these frequencies are statistically quite different for male and female speakers and for different individuals of the same gender. ${ }^{1}$ In gender identification (GID) and speaker identification (SID), the ability to accurately estimate and track speech-related frequencies results in feature distributions with reduced variance and, in principle, provides better identification performance. In vocoding, accurate estimation of speech features results in higher quality speech reproduction and lower coding bit rates. In this paper, we address the problem of isolating and accurately estimating speech components in both time and frequency. While the application in this paper is speech, it should be noted that the methods presented apply equally well to the estimation of any multicomponent nonstationary harmonic signal.

\footnotetext{
${ }^{1}$ The excitation fundamental frequency is normally represented as $F_{0}$. We use the notation $\omega_{0}$ to follow the convention that frequencies are represented by the variations of the Greek " $\omega$ " and representations of the signal are represented by variations of the letter " $f$ ".
}

Cross-spectral methods, based on the short time Fourier transform (STFT) phase, have been recently demonstrated by Nelson to be effective in accurately estimating speech formants and the vocal tract excitation in time and frequency [1]. In that paper, the concept of indicator functions, based on mixed partial phase derivatives, was introduced as a method of isolating regions of the TF surface representing vocal tract resonance and excitation, respectively. The accuracy of the frequency and time estimation provided by the cross-spectral method is based on a phase gradient descent algorithm. The phase gradient represents a second-order statistics, and the indicator functions represent a 4th order statistic. While it may be possible to develop similar methods for nonlinear surfaces, such as the Wigner distribution, cross terms or intermodulation products would likely be a problem. Therefore, the approach taken here is to use the STFT to estimate an initial surface on which the signal components are separated. With this separation, no cross terms are produced in the nonlinear differentiation processes.

We briefly describe an application of cross-spectral methods to the problem of estimating the fundamental of a harmonic signal in time and frequency. We apply the formant resonance indicator functions [1] to de-emphasize the effects of noise and unwanted interference and therefore significantly improve the SNR of the time-frequency representations. We further introduce the correlation spectrum, which is a new method, which features the combined use of an autocorrelation surface representation and a short time Fourier spectral representation of the signal. The correlation spectrum has the important property that it preserves the energy in the 
fundamental of harmonic signals, while greatly attenuating the energy in the higher harmonics. Moreover, the representation is complex, with phase representing the differentiated phase of the short time Fourier transform surface, making it possible to apply the cross-spectral methods developed for the STFT. For speech signals, the correlation spectral representation may be used to determine the excitation fundamental, $\omega_{0}$, unambiguously.

This paper is structured as follows. In Section 2, we briefly describe the structure of speech. Section 3 contains a brief description of the remapping of the STFT surface. In Section 4, we introduce indicator functions computed from the spectral phase, which may be used to identify the properties of signal components which produce the individual STFT surface components. In Section 5, we introduce the correlation spectrum, which combines the properties of the autocorrelation function and the Fourier spectrum. When applied to harmonic signals, the combined representation produces a spectrum with energy at the signal fundamental, while greatly reducing the energy in the harmonics and sub-harmonics. Finally, in Section 6, we present a short experimental example which demonstrates the methods presented in this paper on speech.

\section{THE SIGNAL MODEL}

Speech is an acoustic signal, which results as the vocal tract is excited by a combination of frication and voicing. The primary excitation function is voicing, in which a sequence of pulses is generated at the glottis at the back of the vocal tract. As these pulses excite the vocal tract, the vocal tract resonates at a several resonant frequencies called formants. The formants act as infinite impulse response (IIR) filters, which are quite broad in bandwidth, because of loss in the vocal tract [2]. Because the voiced excitation function is pulsed, its narrowband Fourier representation appears as a harmonic structure

$$
\omega_{n}(t)=n \omega_{0}(t)
$$

where the fundamental excitation frequency $\omega_{0}(t)$ is time dependent. The Fourier spectrum has the representation

$$
F(\omega)=\int_{A}^{B} f(t) h(-t) e^{-i \omega t} d t
$$

where the analysis interval $[A, B]$ is chosen sufficiently large to contain several pulse intervals. In this representation the only spectral energy observed at the output of the mouth is concentrated in the pitch harmonics, which survive the vocal tract (i.e., the pitch harmonics which are within the passband of one of the formants). In trying to estimate $\omega_{0}(t)$, we have the interesting problem that many of the pitch harmonics are severely attenuated. In general, the fundamental, $\omega_{0}(t)$, is not the strongest harmonic and, in fact is frequently completely missing. The normal way of circumventing this problem is to isolate a strong harmonic, estimate the order of the harmonic, and then recover the fundamental by dividing the harmonic frequency by its number. This works, as long as no mistake is made in estimating the harmonic number. If a mistake is made, the results are catastrophic. Our goal is to demonstrate a process which may recover an unambiguous fundamental, even if the fundamental is not present in the speech spectrum. The method we present is based entirely on the phase of the STFT, and uses two applications of the autocorrelation function to reproduce energy at the fundamental and annihilate energy in the harmonics.

\section{CROSS-SPECTRAL METHODS/REMAPPING}

In the methods developed here, we rely heavily on the use of phase derivatives for detecting signal components and estimating their parameters. This is not a new concept. The group delay (GD) and instantaneous frequency (IF) functions appeared in the literature more than 30 years ago (cf. $[3,4]$ ). These functions have the representation

$$
\begin{aligned}
\mathrm{GD}_{f} & =-\frac{d}{d \omega} F(\omega), \\
\operatorname{IF}_{f}(t) & =\frac{d}{d t} \arg \{f(t)\},
\end{aligned}
$$

where the analytic representation of $f(t)$ is assumed in the IF representation, and $F(\omega)$ represents the Fourier transform of the signal $f(t)$.

The STFT is a generalization of the Fourier transform in which the Fourier transforms of the product of the signal $f(t)$ and a sequence of time translations of a (short) analysis window $h(-t)$ are computed. The STFT may therefore be represented as [5]

$$
F(\omega, T)=\int_{-\infty}^{\infty} f(t+T) h(-t) e^{-i \omega t} d t .
$$

While $F(\omega, T)$ is dependent on the windowing function $h(-t)$, we drop the " $h$ " from the notation for simplicity. It can easily be seen that $F\left(\omega_{0}, T\right)$ is the convolution of $f(t)$ and $h(t) e^{i \omega_{0} t}$. For fixed $\omega_{0}, F\left(\omega_{0}, T\right)$ is therefore a (bandpass) filtered version of the original signal.

We define the channelized instantaneous frequency (CIF) and local group delay (LGD) functions as

$$
\begin{aligned}
\operatorname{CIF}_{f}(\omega, T) & =\frac{\partial}{\partial T} \arg \{F(\omega, T)\}, \\
\operatorname{LGD}_{f}(\omega, T) & =-\frac{\partial}{\partial \omega} \arg \{F(\omega, T)\},
\end{aligned}
$$

where we assume that $\arg \{F(\omega, T)\}$ is continuously differentiable.

Group delay and instantaneous frequency have been applied effectively in a number of signal estimation applications. For example, Kay's spectral estimator represents an early use of IF to accurately estimate the frequency of an isolated sine wave in noise [6]. The group delay function has been used quite effectively by Yegnanarayan et al. in spectral estimation and various speech applications (cf. $[7,8])$. These phase derivatives may be estimated by a variety of methods, which are not all equivalent. In Kay's method, the IF is estimated as a simple difference of the phase of the analytic signal and the 
phase of the delayed signal [6]. This method cannot be applied to multi-component signals, since the IF so computed does not necessarily represent the frequency of any of the signal components $[9,10]$. In addition, in Kay's difference method, it is necessary to phase unwrap the estimated IF to compensate for $2 \pi$ phase discontinuities in the differenced phase. Phase derivatives may be estimated as the argument of the product of the analytic signal and the delayed signal (cf. [11]). This eliminates the need to phase unwrap, but still cannot be applied to multi-component signals. The LGD and CIF represent generalizations of the GD and IF functions, and with these generalizations, it is possible to correctly process multi-component signals (cf. $[1,12])$. Sine waves may be estimated accurately using only the CIF. A simple example of this is depicted in Figure 1. However, nonstationary multicomponent signals may be easily processed by using the CIF and LGD functions jointly. The CIF and LGD surfaces may be computed by a number of methods. There is a nice closed form representation computed by Oppenheim and Schafer [13] and used extensively by Yegnanarayana et al. in spectral estimation and various speech applications (cf. $[7,8]$ ). Equally effective is the delay-conjugate-product method presented here (cf. $[1,11,14])$.

To estimate the CIF and LGD surfaces, we define two intermediate cross-spectral surfaces

$$
\begin{aligned}
& \mathrm{C}_{f}(\omega, T, \epsilon)=F\left(\omega, T+\frac{\epsilon}{2}\right) F^{*}\left(\omega, T-\frac{\epsilon}{2}\right), \\
& \mathrm{L}_{f}(\omega, T, \epsilon)=F\left(\omega+\frac{\epsilon}{2}, T\right) F^{*}\left(\omega-\frac{\epsilon}{2}, T\right) .
\end{aligned}
$$

The spectrogram (squared magnitude of the STFT surface), LGD and CIF surfaces may then be estimated as

$$
\begin{aligned}
|F(\omega, T)|^{2} & \approx\left|\mathrm{C}_{f}(\omega, T, \epsilon)\right| \approx\left|\mathrm{L}_{f}(\omega, T, \epsilon)\right|, \\
\operatorname{CIF}_{f}(\omega, T) & \approx \frac{1}{\epsilon} \arg \left\{\mathrm{C}_{f}(\omega, T, \epsilon)\right\}, \\
\operatorname{LGD}_{f}(\omega, T) & \approx-\frac{1}{\epsilon} \arg \left\{\mathrm{L}_{f}(\omega, T, \epsilon)\right\} .
\end{aligned}
$$

Now, we assume that we have a signal which may be written as the (potentially infinite) sum of AM and FM modulated components,

$$
f(t)=\sum_{n} f_{n}(t)
$$

where $f_{n}(t)=A_{n}(t) e^{i\left(\Phi_{n}(t)+\phi_{n}\right)}$, both $A_{n}(t)$ and $\omega_{n}(t)$ are continuous and $\omega_{n}(t)=(d / d t) \Phi(t)$. We would like a method which isolates and simultaneously estimates the locus of each of the signal components $f_{n}$ in time and frequency. To do this, we use the cross-spectral method used by Nelson to estimate instantaneous speech formant frequencies [1]. It was demonstrated that each signal component can be accurately estimated directly from the STFT surface $F(\omega, T)$, as long as the surface satisfies a reasonable local separability condition. Separability is simply the condition that, for any point $\left(\omega_{0}, T_{0}\right)$, at most one signal component can contribute a significant amount of energy to the STFT surface at that

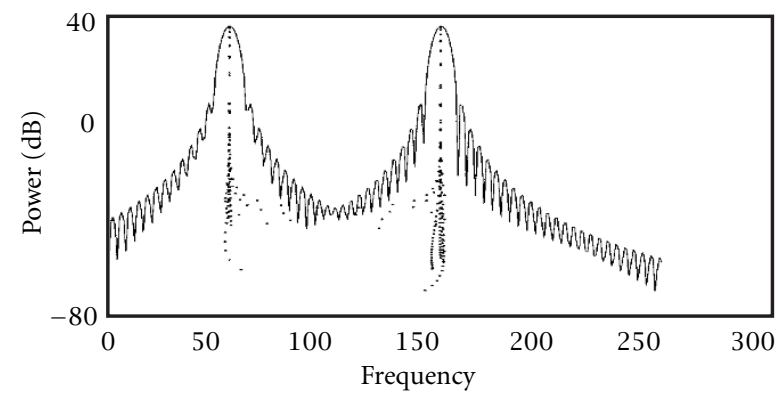

(a)

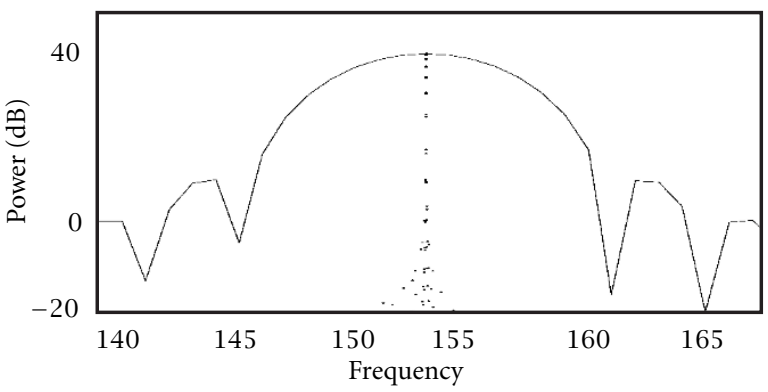

(b)

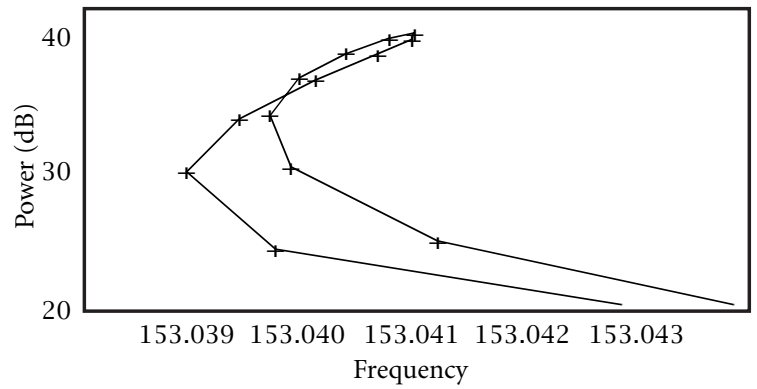

(c)

FIGURE 1: Remapped spectrum of two sine waves computed from a single FFT computed from 128 samples of data Hanning windowed and zero filled to 512 points. Solid line: power spectrum $(\mathrm{dB})$. Dotted line: remapped power spectrum $(\mathrm{dB})$. (a) represents entire spectrum, (b) represents an expansion of the spectral bulge containing the second sine wave, (c) an expansion of the remapped spectrum.

point. Specifically, we assume that $F(\omega, T)$ is separable at $\left(\omega_{0}, T_{0}\right)$, if, for some $n$,

$$
\left|F_{n}\left(\omega_{0}, T_{0}\right)\right|^{2} \gg \sum_{m \neq n}\left|F_{m}\left(\omega_{0}, T_{0}\right)\right|^{2},
$$

where $F_{n}\left(\omega_{0}, T_{0}\right)$ is the STFT of the signal component $f_{n}(t)$.

Separability guarantees that the STFT surface and all of its derivatives locally represent the STFT and the derivatives 
of the strongest signal components. If $F_{n}$ is the dominant signal component at $\left(\omega_{0}, T_{0}\right)$, then $\operatorname{CIF}_{f}\left(\omega_{0}, T_{0}\right)$ provides a re-estimation of the frequency of that component observed at $\left(\omega_{0}, T_{0}\right)$, and $\operatorname{LGD}_{f}\left(\omega_{0}, T_{0}\right)$ represents an estimated timing error of the observation. The relationship

$$
(\omega, T) \longrightarrow\left(\operatorname{CIF}_{f}(\omega, T), T+\operatorname{LGD}_{f}(\omega, T)\right)
$$

provides a remapping of the time-frequency plane, under which separable points on the STFT surface are remapped to points on curves representing the individual FM components of the signal. That is

$$
\operatorname{CIF}_{f}\left(\omega_{0}, T_{0}\right) \approx \operatorname{IF}_{f_{n}}\left(T_{0}+\operatorname{LGD}_{f}\left(\omega_{0}, T_{0}\right)\right)
$$

where $f_{n}$ is the dominant signal component at the separable point $\left(\omega_{0}, T_{0}\right)[1]$. In effect, remapping the surface concentrates the surface components along the curves

$$
\omega_{n}(T)=\operatorname{IF}_{f_{n}}(T)
$$

\section{INDICATOR FUNCTIONS}

The remapping (10) provides a redistribution of the entire TF surface, but the remapping is pointwise, and it is only valid at separable points, where one of the signal components has significant energy. For these points, remapping redistributes the surface energy along the curves (11) representing the functional FM form of the individual signal components. Under remapping, regions of the STFT surface, where the signal has little energy are randomly mapped, resulting in considerable speckled, low energy noise. While it is not always necessary to remove this noise to process the signal, it is generally better to remove it. Since the magnitudes of these unwanted components are generally small, it is possible to use magnitude as a basis for removing some of the noise. However, the signal phase provides a convergence measure which tells us how well the surface components have converged under the remapping. This turns out to be quite useful, since it allows us to construct indicator functions, which measure the attraction of the FM components, which comprise the signal. We present a method, based on mixed partial phase derivatives, which has proven to be very effective in removing much of the surface noise. The use of indicator functions was introduced by Nelson for the purpose of identifying excitation and nearly stationary resonance related STFT components [1]. Our application is slightly different, since we wish to identify STFT components which represent the pitch harmonics $n \omega(t)$, which are never stationary.

The excitation function we wish to estimate is relatively slowly varying. We may expect the rate of change to be less than $500 \mathrm{~Hz} / \mathrm{s}$. If we assume an analysis window of 20 milliseconds, the signal frequency will vary by less than $10 \mathrm{~Hz}$ within the window. We may, therefore, assume that the signal is approximately stationary. We simply note that the mixed partial phase derivative may be represented as

$$
\begin{aligned}
\frac{\partial^{2}}{\partial \omega \partial T} & \arg F_{h}\left(\omega, T_{0}\right) \\
& \approx \frac{1}{\epsilon}\left(\operatorname{CIF}_{f}\left(\omega+\frac{\epsilon}{2}, T_{0}\right)-\operatorname{CIF}_{f}\left(\omega-\frac{\epsilon}{2}, T_{0}\right)\right)
\end{aligned}
$$

for small $\epsilon$. We may assume that surface components near to each other will be attracted to the same signal component under remapping, and, since the signal is assumed to be approximately stationary, we expect the distance between remapped points to be less than the distance between the points before remapping. The mixed partial derivative (13) is, therefore, an indicator of the stationarity of the signal component to which the remapped surface locally converges. If the value of the mixed partial derivative is zero, the signal component may be assumed to be stationary. In testing on many speech files, setting a threshold value of 0.5 resulted in the removal of nearly all of the low energy speckled noise.

\section{CORRELATION AND SPECTRAL REPRESENTATIONS}

Finally, we address the problem of recovery of the pitch fundamental $\omega_{0}$. There are two fundamental problems which must be addressed. Because the vocal tract acts as an infinite impulse (IIR) filter, the only pitch harmonics which are within the passband of one of the formants are observed in the speech spectrum. It is frequently the case that the fundamental pitch frequency and many of the harmonics are not observable in the spectrum. Since we would like to recover the fundamental, we must either estimate the frequency of one of the stronger harmonics and then determine which harmonic it is, or we must somehow reconstruct the pitch fundamental. Misidentification of the harmonic number results in a catastrophic error in the estimated pitch fundamental. To circumvent this problem, Nelson and Wysocki proposed applying the frequency-lag autocorrelation, which is the autocorrelation function applied along the frequency axis of the CIF surface [14]

$$
R_{F}(\Omega, T)=\int_{a}^{b} \operatorname{CIF}_{f}\left(\omega+\frac{\Omega}{2}, T\right) \operatorname{CIF}_{f}^{*}\left(\omega-\frac{\Omega}{2}, T\right) d \omega
$$

where $[a, b)$ is a frequency interval containing one or more formants.

The effect of this application of the autocorrelation function is that a bulge with large energy is produced at the pitch fundamental, since the formant bandwidths are wide enough to contain several pitch harmonics. This process is depicted in Figure 2, and an application to speech is depicted in Figure 3. The problem remains, however, that the pitch fundamental may not be the strongest component in the correlated spectrum. To circumvent this problem, we propose combining two different representations of the signal. The first of these is the frequency correlated CIF surface, which is indexed by time and frequency, and has energy bulges at the pitch harmonics $n \omega_{0}, n=0, \pm 1, \ldots$. The second representation is the time-lag autocorrelation surface

$$
r_{f}(\tau, T)=\int_{-\infty}^{\infty} f\left(t+T+\frac{\tau}{2}\right) f^{*}\left(t+T-\frac{\tau}{2}\right) h(-t) d t
$$




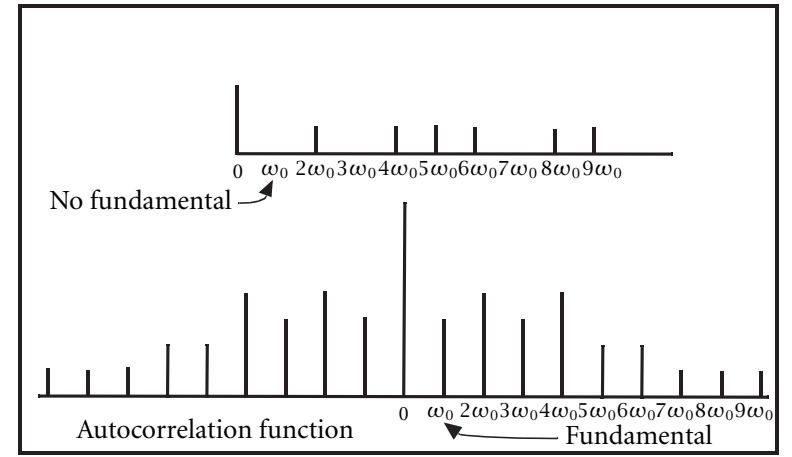

FIGURE 2: Correlation as floating point “GCD”. Top trace: simulated spectrum, with unit energy at the 2nd, 4th, 5th, 6th, 8th, and 9th harmonics. Bottom trace: autocorrelation function with energy at all harmonics.

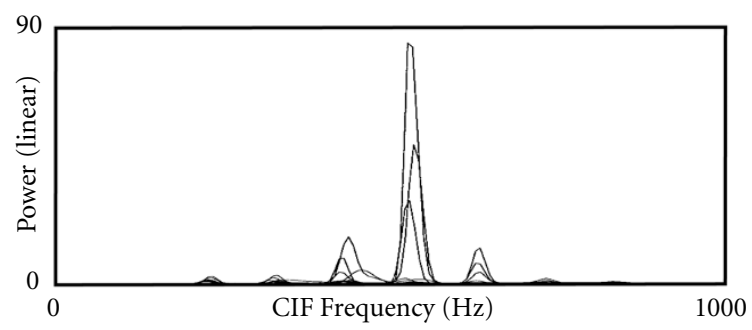

(a)

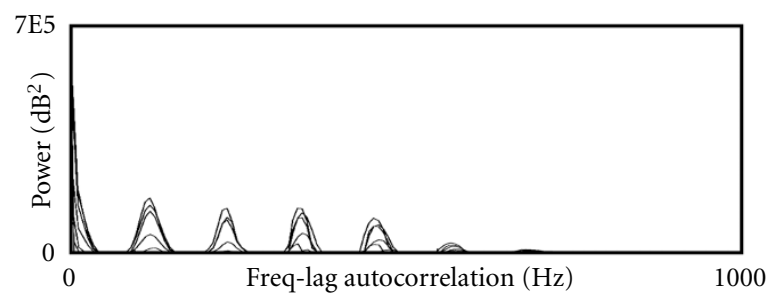

(b)

Figure 3: An application of autocorrelation. (a) Several superimposed power spectra (linear) with missing pitch fundamental. (b) Autocorrelation of CIF components of (a) (dB).

The correlation surface $r_{f}(\tau, T)$ has energy bulges at the pitch fundamental period $P_{0}$ and integer multiples of $P_{0}$. Since $P_{0}$ and $\omega_{0}$ are reciprocals of each other, the representation (15) has energy bulges at $\omega_{0} / n, n= \pm 1, \pm 2, \ldots$. The only "frequency" for which both the frequency and time correlation functions representations of the signal have a common energy bulge is the fundamental $\omega_{0}$.

The question now is, how can we calculate a time correlation surface, which has the same parameterization as the STFT and CIF surfaces. To do this, we start with the CIF sur-

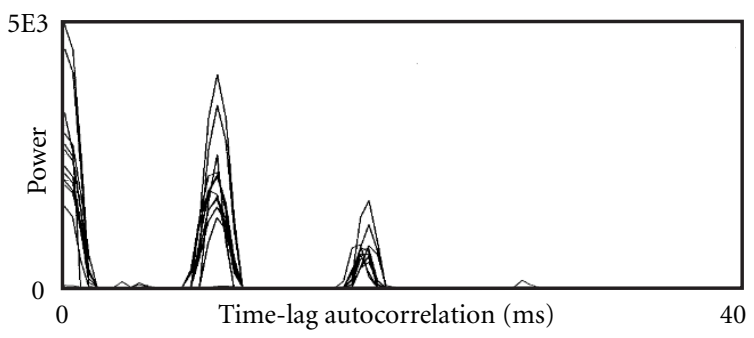

(a)

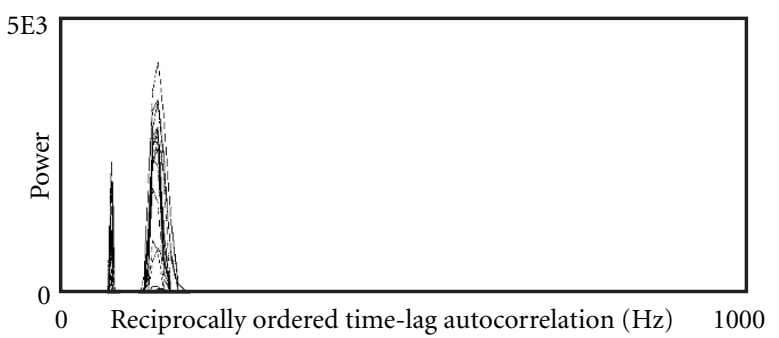

(b)

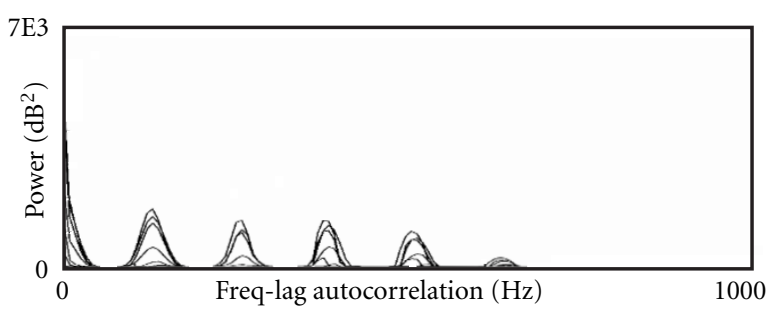

(c)

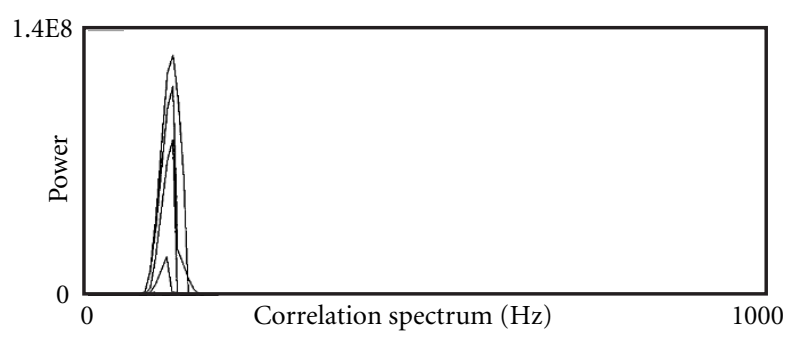

(d)

FIGURE 4: Correlation spectrum calculated from data represented in Figure 3. (a) Time-lag autocorrelation function. (b) Time-lag aurocorrelation function reciprocally ordered. (c) Frequency-lag aurocorrelation function. (d) Correlation spectrum computed as pointwise product of representations (b) and (c).

face, calculate the second derivative indicator function equation (13) and set to zero all CIF components for which the indicator is larger than some $\epsilon$ (e.g., $\epsilon=0.5$ ). We then calculate the frequency correlation surface (14) and estimate the 
TABLE 1: Estimation of two tones in a clean environment.

\begin{tabular}{llcc}
\hline Method & Peak error & $10 \mathrm{~dB}$ BW & $20 \mathrm{~dB}$ BW \\
\hline CS & 0.00039 & 0.0028 & 0.0037 \\
PS & 0.444 & 10 & 13 \\
\hline
\end{tabular}

time autocorrelation surface as

$$
r_{F}(\zeta, T)=\int_{-\infty}^{\infty}\left|R_{F}(\Omega, T)\right|^{2} e^{i \Omega / \zeta} d \Omega, \quad \zeta \neq 0 .
$$

Since period and frequency are reciprocals of each other, the surface $r_{F}(\zeta, T)$ is the time autocorrelation surface indexed by frequency. We may therefore calculate the product surface

$$
\rho_{F}(\Omega, T)=\left|r_{F}(\Omega, T)\right| R_{F}(\Omega, T) .
$$

The function $\rho_{F}(\Omega, T)$ retains the phase of the surface $R_{F}(\Omega, T)$ and is expected to have a bulge of energy at $\Omega=\omega_{0}$, but the energy in the harmonics are suppressed by the factor $r_{F}(\Omega, T)$, and the subharmonics are suppressed by the factor $R_{F}(\Omega, T)$. Experimentally, the combined representation $\rho_{F}(\Omega, T)$ resulted in a 12 to $15 \mathrm{~dB}$ improvement in harmonic/sub-harmonic suppression. An example of this process applied to speech is depicted in Figure 4.

\section{EXPERIMENTAL RESULTS}

The methods presented here have been applied to synthesized signals and many segments from speech databases. As a simple example of the remapping, a signal consisting of two sine waves was processed with a 257 long Hanning window, zero filled to 1024 samples. If we assume that the resolution, due to frequency quantization of the transform is unity, the average error in the remapped estimate was 0.00039 , while the error in estimating the frequency as the index of the coefficient of the power spectrum with the largest magnitude was 0.444 , an accuracy improvement factor of 1000, as depicted in Figure 1. The error in estimating the signal frequency by peak picking the powerspectrum and the remapped cross-spectrum are presented in Table 1, with the respective bandwiths of the two methods measured at $10 \mathrm{~dB}$ and $20 \mathrm{~dB}$ below peak signal power.

The process was applied to 412 files of data from the SWITCHBOARD database, nearly evenly split between male and female. The data represent actual telephone conversations, 8-bit mu-law encoded and sampled at $8 \mathrm{kHz}$. The STFT surfaces were computed using a 513 sample Hanning window and a zero-filled 1024 point transform. The CIF and LGD surfaces were computed using 1 sample delays in time and frequency, respectively. The STFT surfaces were remapped, and the noise speckle was removed with the indicator function described above, with a threshold of $\epsilon=0.5$. The fundamental was re-inserted using the frequency-lag autocorrelation function, and the fundamental was recovered unambiguously by combining the time-lag autocorrelation function and the spectrum, augmented by the frequency-lag autocorrelation. The spectrograms of two samples of speech are represented

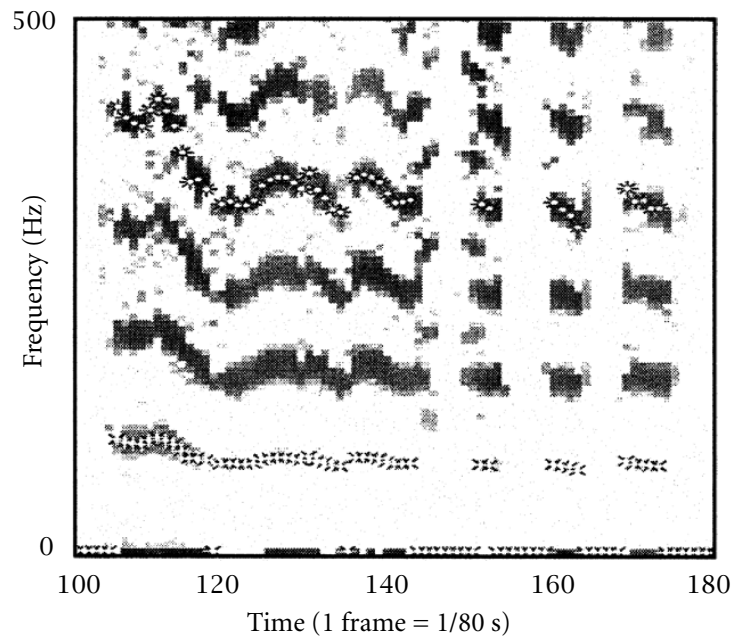

FIGURE 5: Spectrogram of speech with missing pitch fundamental. For each frame, one star with a white center is displayed at the fundamental and 4 th harmonic.

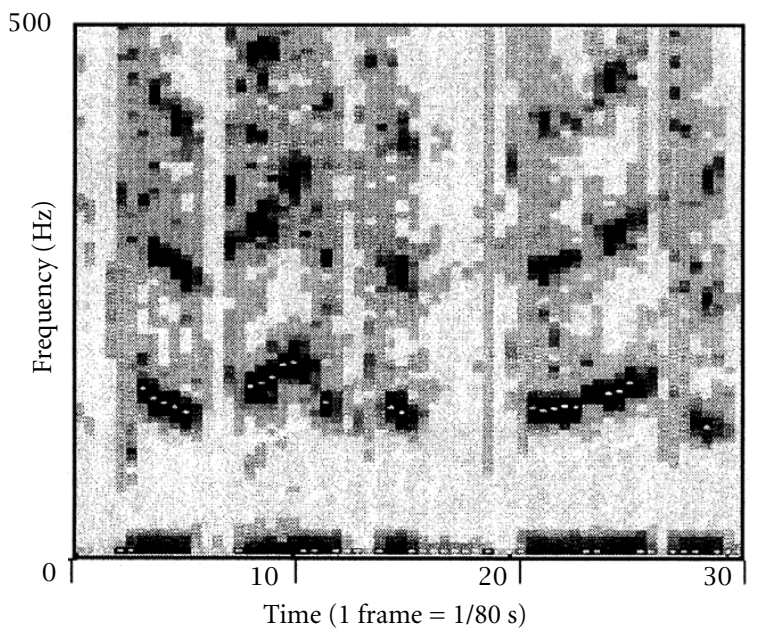

FIGURE 6: Spectrogram of noisy speech. For each frame, one star is displayed at the estimated fundamental.

in Figures 5 and 6. In Figure 5, the fundamental is missing in the spectrogram, but was recovered correctly in processing. In this display, the fundamental and 4th harmonic are superimposed on the spectrogram as black stars with white centers. For each 1/80 second frame of data, one such pair of stars is displayed. In Figure 6, speech at about $10 \mathrm{~dB}$ SNR was processed. The pitch fundamental is displayed as white stars. The performance of the presented displays is representative of the process. In each case the frequency fundamental was estimated as the argument of the component with the largest magnitude on the surface representing the product of the time and frequency lag correlation surfaces. The process has been applied to a synthesized typical HF environment, representing a mistuned single sideband (SSB) speech signal in noise, with automatic gain control (AGC). The process correctly detected the signal and resulted in the correct estimation of the fundamental. 


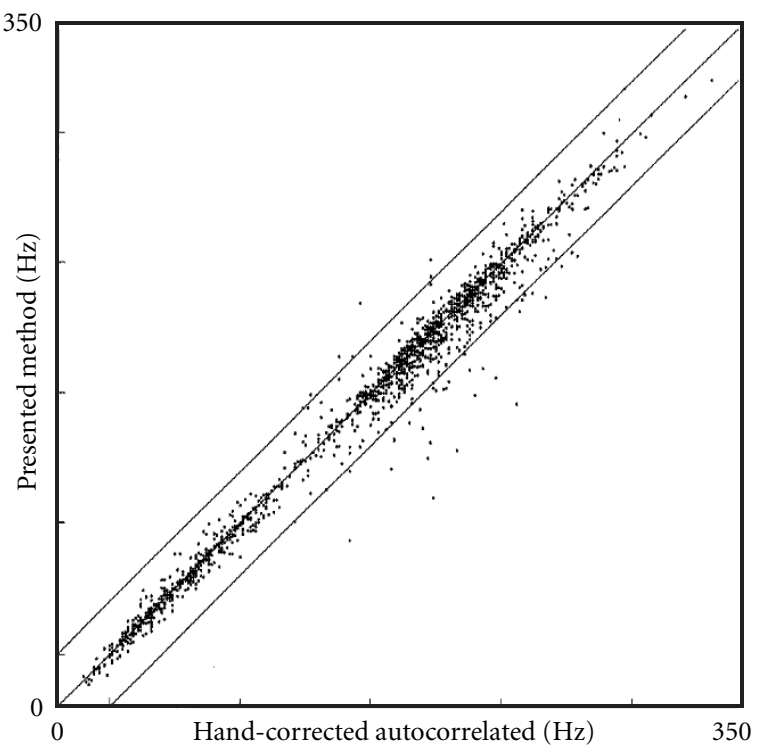

FIGURE 7: Comparison of pitch estimated using the methods of this paper and hand-corrected autocorrelation-based estimates. Diagonal represents perfect agreement. Two solid lines represent $\pm 20 \mathrm{~Hz}$.

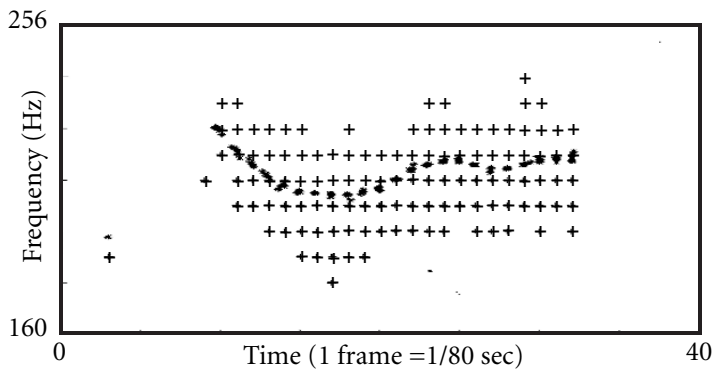

FIGURE 8: Remapping of individual TF points on a lattice with time resolution $1 / 80$ second and frequency resolution $8 \mathrm{~Hz}$. (+: original points, $*$ : remapped points).

\section{CONCLUSIONS}

Cross-spectral methods provide a simple method for accurately estimating multi-component FM signals, and the use of the mixed partial indicator function effectively removes noise speckle. By combining representations of the frequency-lag and time-lag autocorrelation functions, has been demonstrated that it is possible to reconstruct and isolate the harmonic fundamental, even if it is absent from the original spectrum. The method has been implemented in MATLAB on both a SUN workstation and a pentium $400 \mathrm{Mhz}$ PC. On both machines, the MATLAB implementations are slightly faster than real time. The method was compared on more than 1400 vowels to pitch estimates published by Hillenbrand et al. [15], which were made by first applying a correlation based method and then hand editing the correlation based estimates. No attempt was made to modify the estimates made by the process presented here. The standard deviation of the differences between estimates made the two ways is $7.14 \mathrm{~Hz}$. The two methods are in close agreement, as depicted in Figure 7.
Finally, Figure 8 demonstrates the improved accuracy of the presented method over the STFT. The lattice of STFT points (time resolution $=1 / 80$ second and frequency resolution $=8 \mathrm{~Hz}$ ) surviving the indication test are displayed as +'s and the remapped points superimposed as $*$ 's.

\section{REFERENCES}

[1] D. J. Nelson, "Cross-spectral methods for processing speech," J. Acoust. Soc. Amer., vol. 110, no. 5, pp. 2575-2592, 2001.

[2] J. L. Flanagan, Speech Analysis Synthesis and Perception, Springer-Verlag, Berlin, 2nd edition, 1972.

[3] J. B. Thomas, An Introduction to Statistical Communication Theory, Wiley, New York, 1969.

[4] A. J. Gibbs, "The design of digital filters," Australian Telecommunication Research Journal, vol. 4, pp. 29-34, 1970, reprinted in Digital Signal Processing, eds. L. R. Rabiner and C. M. Rader, IEEE Press, New York, pp. 35-42, 1972.

[5] D. Gabor, "Theory of communication," in Proc. of the IEE, vol. 93, pp. 429-457, 1946.

[6] S. M. Kay, "Statistically/computationally efficient frequency estimation," in Proc. IEEE Int. Conf. on ASSP, pp. 2292-2295, New York, 1988.

[7] P. S. Murthy and B. Yegnanarayana, "Robustness of groupdelay-based method for extraction of significant instants of excitation from speech signals," IEEE Trans. on Speech and Audio Processing, vol. 7, no. 6, pp. 609-619, 1999.

[8] P. S. Murthy and B. Yegnanarayana, "Formant extraction from phase using weighted group delay function," in Electronics Letters, vol. 25, pp. 1609-1611, 1989.

[9] L. Cohen, "Time-frequency distributions-a review," in Proc. of the IEEE, vol. 77, pp. 941-981, 1989.

[10] L. Cohen, Time-Frequency Analysis, Prentice-Hall, Englewood Cliffs, NJ, 1995.

[11] D. J. Nelson, "Special purpose correlation functions for improved signal detection and parameter estimation," in Proc. IEEE Int. Conf. on ASSP, pp. 73-76, April 1993.

[12] S. Umesh and D. J. Nelson, "Computationally efficient estimation of sinusoidal frequency at low SNR," in Proc. IEEE Int. Conf. on ASSP, vol. 5, pp. 2797-2800, May 1996.

[13] A. V. Oppenheim and R. W. Schafer, Digital Signal Processing, Prentice-Hall, Englewood Cliffs, NJ, 1975.

[14] D. J. Nelson and W. Wysocki, "Cross-spectral methods with an application to speech processing," in Proc. of the SPIE Adv. Sig. Proc. Conf., July 1999.

[15] J. M. Hillenbrand, L. A. Getty, M. J. Clark, and K. Wheeler, "Acoustic characteristics of American English vowels," J. Acoust. Soc. Amer., vol. 97, no. 5, pp. 3099-3111, 1995.

Douglas Nelson was born in Minneapolis, Minnesota on 5 November 1945. He received a bachelor degree in Mathematics from the University of Minnesota in 1967 and a doctorate in Mathematics from Stanford University in 1972. After spending three years as an assistant professor at CarnegieMellon University, he accepted a position at the National Security Agency at Fort Meade, Maryland, where he has been from 1975 to the present. At the NSA, Dr Nelson's primary interest has been developing signal processing algorithms for radar, communications, and speech related problems. Several of these algorithms have been patented or have patents pending. 